
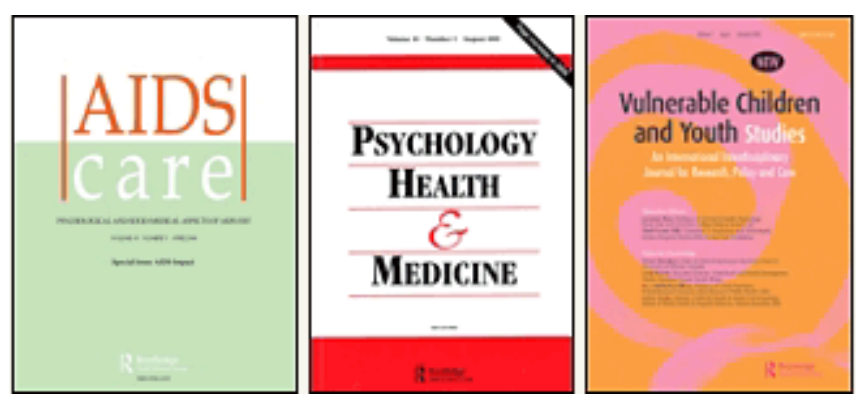

\title{
Suicide attempts among people living with HIV in France
}

\begin{tabular}{|r|l|}
\hline Journal: & $\begin{array}{l}\text { AIDS Care - Psychology, Health \& Medicine - Vulnerable Children } \\
\text { and Youth Studies }\end{array}$ \\
\hline Manuscript ID: & AC-2007-04-0173.R1 \\
\hline Journal Selection: & AIDS Care \\
\hline Keywords: & suicide attempted, discrimination, side effects, lipodystrophy \\
\hline \multicolumn{2}{|r}{} \\
\hline
\end{tabular}

\section{s) ScholarONE" \\ Manuscript Central}




\section{Suicide attempts among people living with HIV in France}

Marie Préau ${ }^{3,1,2}$, Anne-Déborah Bouhnik ${ }^{1,2}$, Patrick Peretti-Watel ${ }^{1,2}$, Yolande Obadia ${ }^{2,1}$, Bruno Spire ${ }^{1,2}$ and the VESPA Group

${ }^{1}$ INSERM Research Unit 379 "Epidemiology and Social Sciences applied to medical innovation", Marseilles, France

${ }^{2}$ Southeastern Health Regional Observatory (ORS-PACA), Marseilles, France

${ }^{3}$ LabECD, Psychology department, Nantes University, NANTES, France

Corresponding author: Marie PRÉAU

INSERM U379, 23 rue Stanislas Torrents, 13006 MARSEILLE, France

LABCD, Psychology department, Nantes University, NANTES, France

ㄹ: +33240141304 : +33240141255

@: preau@marseille.inserm.fr 


\section{Suicide attempts among people living with HIV in France}

Objectives: This study examined the prevalence and characteristics of attempted suicide (AS) among a representative sample of French Human Immunodeficiency virus (HIV) infected individuals.

Method: In 2003, a face-to-face survey was conducted among people living with HIV (PLWHA) selected in a random, stratified sample of French hospital departments. Among solicited individuals, 2,932 agreed to participate and were asked if they had ever AS.

Results: Among the respondents, 23\% had AS. Female gender, younger age, native French citizenship, reporting household financial difficulties, having been HIV-contaminated through homosexual contact or through IDU and suffering from lipodystrophy-related symptoms were all independently associated with AS. HIV-discrimination and the lack of social support from family remained independently associated with AS.

Conclusions: The findings indicate a high level of AS among PLWHA and emphasize the multiple roles of factors associated with living with HIV, together with socio-demographic factors. Our results enable the possibility for vulnerable groups to be targeted for specific future interventions in order to prevent AS.

Key Words: suicide attempt, discrimination, side effect, lipodystrophy 


\section{Suicide attempts among people living with HIV in France}

\section{INTRODUCTION}

Suicide ideation and psychological morbidity among people living with HIV/AIDS (PLWHA) has been an important subject in the context of HIV infection. Previous studies have reported that two-thirds of PLWHA have suicidal ideation at some point. Half of them reporting suicide plans and one quarter suicide attempts. In one study, a third of PLWHA reported suicidal ideation (Robertson, Parsons, Van Der Horst, \& Hall, 2006). The majority of studies of suicidal ideation have compared HIV infected and non-HIV infected populations. These studies allow researchers to determine the prevalence of suicide ideation but do not clarify the reasons associated with suicidal ideation, which still need to be understood. Moreover, studies of HIV-infected individuals date prior to 1996 and the availability of highly active antiretroviral therapy. AIDS is a paradigm of a medical illness which requires a biopsychosocial approach and research on psychiatric morbidity and mortality needs to be promoted in order to unravel the bio-psychosocial determinants of mental health (Cohen, 1990). Moreover, in the specific context of HIV infection, the impact of discrimination behavior needs to be described and understood. HIV-related stigmatization remains a potent stressor for PLWHA (Dias, Matos, \& Goncalves, 2006; Greene \& Banerjee, 2005; PerettiWatel, Spire, Pierret, Lert, \& Obadia, 2006; Vanable, Carey, Blair, \& Littlewood, 2006) that can have an impact on suicidal ideation.

This study examined the prevalence of suicide attempts (SA) among a representative sample of French PLWHA and aimed at identifying the bio-psychosocial characteristics of those who attempted suicide. 


\section{METHODS}

\section{Data Collection}

In 2003, the French National Agency for AIDS Research (ANRS, France) supported a national cross-sectional survey conducted among a random stratified sample of PLWHA, recruited at 102 French hospital departments delivering HIV care. Its aim was to describe the living and socio-economics conditions of PLWHA in France. The stratification criteria were the hospital departments' geographic location and the HIV caseload (Peretti-Watel et al., 2005). Eligible subjects were outpatients at least 18 years of age and who had been diagnosed with HIV-1 at least 6 months previously. Those who were foreign citizens had to have been living in France for at least six months to be eligible. At each participating hospital, participation in the survey was proposed to each eligible outpatient at the end of their consultation, whether they were attending a regular or a special HIV related appointment. The participants gave their written informed consent and were each compensated with a 15 -euro voucher after the study (patients were not aware of this payment when asked to participate).

Among 4,963 eligible individuals, 2,932 participants were included in the study. Two hundred and sixty four were not solicited because of major cognitive impairment or health problems, while 1,767 individuals declined to participate. Individuals who refused most frequently cited a lack of time; compared to participants, they were more likely to have been HIV-infected other than through homo/bisexual contacts and to be employed at the time of study.

\section{Weighting procedure}

Overall, non-participation reached $41 \%$ of the randomized individuals, with substantial differences according to individuals' HIV transmission category, employment status and immunological status. Major socio-demographic and health-related characteristics were 
collected both among respondents and non-respondents, allowing for a weighting procedure to take into account this participation bias. This weighting procedure included two steps: firstly, considering the unequal probability of enrolment, related to the heterogeneous frequency of participants' hospital visits, a weight was attributed to each individual corresponding to the inverse number of hospital visits he (she) had reported for the preceding year. Secondly, to account for non-response, an additional weight was computed, using a method of calibration adjustment (Deville \& Särndal, 1992), in such a way that the weighted distribution of the participants regarding transmission group, employment status and immunological status was comparable to that of the entire eligible population.

\section{Questionnaires}

Individuals who agreed to participate answered a face-to-face questionnaire (398 closedended questions) administered by a trained interviewer using the CAPI (Computer Assisted Personal Interview) system. This questionnaire dealt with a range of aspects of the daily life of PLWHA and their socio-economic conditions. In parallel, the consulting physician completed a short medical questionnaire.

\section{Variables}

\section{Suicide attempts}

Participants were asked whether they had ever attempted suicide, either before or after HIV infection: once/several times/never. Those individuals having attempted suicide one or more times were compared with those who had never attempted suicide.

\section{HIV-discrimination}


Participants were consecutively asked whether they had ever felt discriminated against due to their serostatus (yes/no) by their relatives, by their friends, by sexual partners, or at work. A score of HIV-discrimination was computed by summing up the answers to these 4 questions $\left(\right.$ yes $=1 /$ no $\left.^{\prime}=0\right)$.

\section{Sociodemographic characteristics}

Sociodemographic characteristics included age, gender, nationality, history of incarceration, employment status and an appreciation of the household financial situation (satisfactory/difficult/very difficult).

\section{Social support}

The frequency of contacts with relatives during the prior 12 months was evaluated using a 5point scale: at least once a week / at least once a month / at least once every three months / at least once every 6 months / never. Regular contact with relatives was defined as reporting contact at least once a week. Regular contact with friends was defined in the same way.

\section{Perception of treatment side-effects}

Participants were asked whether they perceived the side-effects of their treatment as nonexistent / not disturbing / fairly disturbing / rather disturbing or very disturbing. Another question dealt with the presence of lipodystrophy-related symptoms.

\section{Substance use in the prior 12 months}

Participants were asked whether they had used anxiolytics and/ or antidepressants during the previous 12-month period. Since alcohol is commonly consumed, only binge drinking 
(defined as taking at least 6 alcoholic drinks on a single occasion) was taken into consideration.

\section{Medical characteristics}

The medical questionnaire included information about each individual's HIV-transmission group, the then current viral load and CD4 cell count, CD4 cell count at treatment initiation, clinical stage, time of diagnosis and HIV-treatment.

\section{Statistical Analysis}

Chi-squared test and Student's t-test were used to compare participants who had attempted suicide with the rest of the sample. To identify the factors independently associated with suicide attempt, logistic models were used. A stepwise procedure was used here to select statistically significant factors in a multivariate model (entry threshold $\mathrm{p}<0.10$ ). Statistical analyses were performed using the SPSS v 12.0.1 software program (SPSS, Inc., Chicago, IL, U.S.A.).

\section{RESULTS}

There were 2,932 participants in our survey. Women accounted for 29\%, medium age was 41 $(\mathrm{IQR}=[36-47])$ and one in five patients was an immigrant. Characteristics regarding sociodemographics as well as medical characteristics of these participants are provided in table 1. As far as HIV-discrimination is concerned, $12 \%$ of individuals reported having felt discriminated against by their relatives, $12 \%$ by their friends, $7 \%$ at work and $17 \%$ by sexual partners. Using the score of HIV-discrimination, 34\% reported at least one sphere of discrimination. It appears that HIV transmission group and discrimination are significantly associated $(\mathrm{p}<0.001)$, with $44 \%$ of participants HIV-infected by drug use reporting at least 
one sphere of discrimination, while this value was $34 \%$ for participants contaminated by homosexual contact, $28 \%$ by heterosexual contact and $23 \%$ by others routes.

Two-thirds of individuals had regular contact with their relatives in the prior twelve months and three quarters had had contact with their friends.

Consumption of antidepressants was reported by $10 \%$ of individuals and $21 \%$ had consumed anxiolytics in the prior twelve months. Regarding alcohol consumption, 24\% reported that they had indulged in binge drinking at least once a month during the previous 12 months.

\section{Suicide attempts (SA)}

In our survey, $22 \%$ of participants had attempted suicide at least once during their life.

Those who had attempted suicide were more likely to be individuals born in France, women, younger adults, those with a lower level of education, unemployed participants and those reporting a difficult or a very difficult household financial situation (table 1). Having a history of incarceration was associated with SA and when considering the mode of HIV-acquisition, homosexual men and injecting drug users were significantly associated with higher frequencies of suicide attempts. Regarding HIV-diagnosis, individuals who had been diagnosed for a longer period of time were also associated with higher frequencies of SA.

Regarding social support, reporting no regular contact with friends and especially no contact with relatives was associated with SA.

Consumption of anxiolytics and consumption of antidepressants were associated with higher levels of SA $(32 \%$ versus $19 \%-p<0.001$ and $37 \%$ versus $18 \%-p<0.001$ respectively). Binge drinking also tended to be associated with SA, as $24 \%$ of individuals who had indulged in binge drinking at least once a month in the previous 12 months had attempted suicide versus $21 \%$ of those who had not $(p=0.060)$. 
In addition, reporting having suffered from HIV-discrimination by family, friends, at work or by sexual partners were all associated with SA. More precisely, the level of SA was $44 \%$ among those HIV-discriminated by relatives (versus $18 \%$ - $p<0.001$ ) $42 \%$ among HIVdiscriminated by friends (versus 19\% - p<0.001), 36\% among HIV-discriminated at work (versus $20 \%-\mathrm{p}<0.001$ ) and $32 \%$ among those HIV-discriminated by sexual partners (versus $20 \%-\mathrm{p}<0.001)$. The score of HIV-discrimination was also strongly associated with SA.

With respect to clinical variables, individuals with undetectable viral load as well as those with CD4 cell count above 200 reported significantly lower rates of SA. By contrast, neither being at $\mathrm{C}$-stage of the illness nor having a lower mean CD4 cell count at treatment initiation, were associated with SA.

Repercussions of HIV-treatment were strongly associated with suicide. Those who had attempted suicide were more likely to report side-effects as disturbing or very disturbing and more often reported lipodystrophy-related symptoms.

Table 2 shows the result of the multivariate model identifying independent factors associated with antecedent of SA. Factors positively associated with antecedent of SA were: being under 50 years of age, being a woman, reporting household financial difficulties, having been HIVcontaminated through homosexual contact or through IDU and suffering from lipodystrophyrelated symptoms. The factors diminishing the likelihood of AS were: being an immigrant and having regular family contact. In addition to all these factors, the HIV-discrimination score remained significantly and independently associated with SA.

\section{DISCUSSION}


Suicide attempts appear to be highly prevalent among PLWHA in France since one in five individuals living with HIV has attempted suicide. This is much higher than the prevalence observed in the adult French general population which is $8 \%$ (Ministère de l'Emploi de la cohésion sociale et du logement \& Solidarités, 2006).

More specifically, in France $6 \%$ of males and $9 \%$ of females in the general population have attempted suicide. However, in our representative sample of HIV-infected patients, these figures were $19.5 \%$ of males and $26.7 \%$ of females. Comparison of these results highlights the extent of the problem of suicide attempts among the HIV-infected population.

SA were associated with several types of bio-psychosocial characteristics, age and gender, financial precariousness but also sexual orientation, a history of drug use, bodily changes due to HIV infection and last, but not least, social integration and acceptation in terms of discrimination and family social support.

Before discussing our results, we must acknowledge several limitations in the present study. Firstly, our study included cross-sectional data; using longitudinal data would have been more appropriate in order to understand the causality between different factors. Secondly, there was no precision regarding the date of the SA, therefore, there is no indication of whether they occurred before or after HIV-diagnosis, which consequently restricts our interpretations.

In addition, our study shares with many others, some of the general methodological problems related to self-report assessment based on participants' declarations, which may be affected by a social desirability bias. Nevertheless, this VESPA study should be considered as a valuable complement to existing longitudinal data, because it is the first survey carried out in France of a large and representative random sample of HIV-infected outpatients. Last but not least, there is a lack of data about prevalence of psychiatric disorders in this sample. The design of the study did not take into account information about the psychopathology of PLWHA reporting 
AS. This limitation is important due to the fact that literature on the subject has identified some psychopathological conditions such as post traumatic disorder or psychiatric disorder as being more likely associated with AS PLWHA who are likely to need particular psychiatric assessment, medical and mental health treatment (Ayuso Mateos, Lastra, \& Montanes, 1996; Nina A. Cooperman \& Jane M. Simoni, 2005; James, Rubin, \& Willis, 1991; Lewis, 2005; Roy, 2003). Nevertheless, this study aimed at identifying factors related to serostatus associated with AS in order to give some guidelines related to improving the follow-up of HIV infected people.

The experience of discrimination seems to weigh heavily in regard to SA. Our study shows a high prevalence of discrimination against HIV as one in three individuals questioned had encountered it. Experience of discrimination attitudes has already been described (Dias, Matos, \& Goncalves, 2006; Vanable, Carey, Blair, \& Littlewood, 2006) and has been shown to be associated with mental health, impaired quality of life and non-adherence (Peretti-Watel, Spire, Pierret, Lert, \& Obadia, 2006; Preau et al., 2007; Rintamaki, Davis, Skripkauskas, Bennett, \& Wolf, 2006). Although we could not state that SA occurred after HIV-diagnosis, our results show that individuals having attempted suicide were more likely to have experienced discrimination attitudes related to sexual orientation, drug consumption and to the particularities of HIV infection (de Graaf, Sandfort, \& Ten Have, 2006; Huebner, Rebchook, \& Kegeles, 2004; Mills et al., 2004; Warner et al., 2004). The consequences in terms of AS are important, insofar as that these various vulnerabilities can be combined together for certain PLWHA, and this may increase the possibility of AS.

Those participants who had attempted suicide more frequently reported lipodystrophy syndrome. This result can be interpreted in terms of the non-acceptance of body image which 
can lead to mental disorders and which may also lead to the fear of stigmatization. Authors have shown that because lipodystrophy results in shape modification, it can be a visibly external sign of HIV and so can be perceived by those with the illness as a source of stigmatization (Goldin, 1994; Persson, 2005). These results emphasize the need to develop several types of interventions.

They also emphasize the need to develop several types of interventions. Firstly, at the level of patient-doctor interaction, there is a need for lipodystrophy syndrome to be taken into account during the follow-up of PLWHA. Secondly, among the general population, it seems relevant to explore the patterns of illness visibility. It would be of interest to investigate these patterns in the framework of social sciences, in order to understand their connotations, i.e. in terms of their social significance and implications in terms of current general practices. Social and ethical implications are also important with specific attention to be developed in educational and media representations of HIV.

The determinant role of contact with family, a result already known, needs to be underlined in the specific context of HIV infection where discriminatory behaviors are frequent and where the role of family contact should be highlighted (Compton, Thompson, \& Kaslow, 2005; Heikkinen, Aro, \& Lonnqvist, 1994; Suppapitiporn, Thavichachart, \& Suppapitiporn, 2004; Thompson, Kaslow, Short, \& Wyckoff, 2002).

This study found that those who attempt suicide are more frequent among participants who have been living with HIV for a longer period of time. However, this factor was not found in the multivariate model after adjustment for discrimination experience. This suggests that the first generation of participants is likely to have had more exposure to discrimination, but also 
that discriminatory behavior is still a factor which propagates suicide attempts even when taking into account patient generation heterogeneity.

In addition, these results confirm that HIV-infected people present specific vulnerability to SA as previously found in such groups as drug addicts (Haller \& Miles, 2003), people concerned by stigma due to homosexual orientation (Courtenay-Quirk, Wolitski, Parsons, \& Gomez, 2006; Grossman, 1994; Schneider, Taylor, Hammen, Kemeny, \& Dudley, 1991) and people concerned by social vulnerability (N. A. Cooperman \& J. M. Simoni, 2005).

The results suggest that suicide prevention measures should be implemented for each specific kind of vulnerability. Interventions should target individuals who have the different factors associated with suicide attempts in this representative sample of PLWHA. In addition, policy makers need to pay attention to discrimination of people living with HIV and make efforts to propagate better acceptance of HIV disease in society. 


\section{ACKNOWLEDGEMENTS}

Special thanks to the members of the VESPA Group: C. Afsa (INSEE), A. Bonnet (UFR Psychopathologie, Universite' de Provence), A.D. Bouhnik (INSERM U379/ORS PACA), V. Di Paola (LEST), R. Dray-Spira (INSERM U88), J. Fagnani (CNRSUMR Matisse), L. Fernandez (UFR Psychopathologie, Universite' de Provence), I. Heard (INSERM U430), F. Lert (INSERM U88), Y. Obadia (ORS PACA/INSERM U379), J.L. Pedinielli (UFR Psychopathologie, Universite' de Provence), P. Peretti-Watel (ORS PACA/INSERM U379), J. Pierret (CERMES-INSERM U504-UMR 8559), B. Riandey (INED), M.A. Schiltz (CERMES-INSERM U504-UMR 8559), R. Sitta (INSERM U88), and B. Spire (INSERM U379/ORSPACA).

This study was supported by the French National Agency of AIDS Research (ANRS, France). 


\section{REFERENCES}

Ayuso Mateos, J. L., Lastra, I., \& Montanes, F. (1996). Research in psychopathology in patients with HIV/AIDS and psychiatric services in Spain. AIDS Care, 8(2), 233-239.

Cohen, M. A. (1990). Biopsychosocial approach to the human immunodeficiency virus epidemic. A clinician's primer. Gen Hosp Psychiatry, 12(2), 98-123.

Compton, M. T., Thompson, N. J., \& Kaslow, N. J. (2005). Social environment factors associated with suicide attempt among low-income African Americans: the protective role of family relationships and social support. Soc Psychiatry Psychiatr Epidemiol, 40(3), 175-185.

Cooperman, N. A., \& Simoni, J. M. (2005). Suicidal Ideation and Attempted Suicide Among Women Living With HIV/AIDS. Journal of Behavioral Medicine, 28(2), 149-156.

Cooperman, N. A., \& Simoni, J. M. (2005). Suicidal ideation and attempted suicide among women living with HIV/AIDS. J Behav Med, 28(2), 149-156.

Courtenay-Quirk, C., Wolitski, R. J., Parsons, J. T., \& Gomez, C. A. (2006). Is HIV/AIDS stigma dividing the gay community? Perceptions of HIV-positive men who have sex with men. AIDS Educ Prev, 18(1), 56-67.

de Graaf, R., Sandfort, T. G., \& Ten Have, M. (2006). Suicidality and Sexual Orientation: Differences Between Men and Women in a General Population-Based Sample From The Netherlands. Arch Sex Behav.

Deville, J.-C., \& Särndal, C.-E. (1992). Calibration estimation in survey sampling. J Am Stat Assoc, 87(418), 375-382.

Dias, S. F., Matos, M. G., \& Goncalves, A. C. (2006). AIDS-related stigma and attitudes towards AIDS-infected people among adolescents. AIDS Care, 18(3), 208-214.

Goldin, C. S. (1994). Stigmatization and AIDS: critical issues in public health. Soc Sci Med, 39(9), 1359-1366.

Greene, K., \& Banerjee, S. C. (2005). Disease-Related StigmaComparing Predictors of AIDS and Cancer Stigma. J Homosex, 50(4), 185-209.

Grossman, A. H. (1994). Homophobia: a cofactor of HIV disease in gay and lesbian youth. $J$ Assoc Nurses AIDS Care, 5(1), 39-43.

Haller, D. L., \& Miles, D. R. (2003). Suicidal ideation among psychiatric patients with HIV: psychiatric morbidity and quality of life. AIDS Behav, 7(2), 101-108.

Heikkinen, M., Aro, H., \& Lonnqvist, J. (1994). Recent life events, social support and suicide. Acta Psychiatr Scand Suppl, 377, 65-72.

Huebner, D. M., Rebchook, G. M., \& Kegeles, S. M. (2004). Experiences of harassment, discrimination, and physical violence among young gay and bisexual men. Am J Public Health, 94(7), 1200-1203.

James, M. E., Rubin, C. P., \& Willis, S. E. (1991). Drug abuse and psychiatric findings in HIV-seropositive pregnant patients. Gen Hosp Psychiatry, 13(1), 4-8.

Lewis, C. F. (2005). Post-traumatic stress disorder in HIV-positive incarcerated women. $J$ Am Acad Psychiatry Law, 33(4), 455-464.

Mills, T. C., Paul, J., Stall, R., Pollack, L., Canchola, J., Chang, Y. J., et al. (2004). Distress and depression in men who have sex with men: the Urban Men's Health Study. Am J Psychiatry, 161(2), 278-285.

Ministère de l'Emploi de la cohésion sociale et du logement, \& Solidarités, M. d. 1. S. e. d. (2006). Suicide and suicidal attemted in France, studies and results, $n^{\circ} 468$. 
Peretti-Watel, P., Riandey, B., Dray-Spira, R., Bouhnik, A. D., Sitta, R., \& Obadia, Y. (2005). Comment enquêter la population séropositive en France ? L'enquête ANRS-EN12VESPA 2003. Population, 60(4), 525-550.

Peretti-Watel, P., Spire, B., Pierret, J., Lert, F., \& Obadia, Y. (2006). Management of HIVrelated stigma and adherence to HAART: evidence from a large representative sample of outpatients attending French hospitals (ANRS-EN12-VESPA 2003). AIDS Care, $18(3), 254-261$.

Persson, A. (2005). Facing HIV: body shape change and the (in)visibility of illness. Med Anthropol, 24(3), 237-264.

Preau, M., Marcellin, F., Carrieri, M. P., Lert, F., Obadia, Y., \& Spire, B. (2007). Healthrelated quality of life in French people living with HIV in 2003: results from the national ANRS-EN12-VESPA Study. Aids, 21 Suppl 1, S19-S27.

Rintamaki, L. S., Davis, T. C., Skripkauskas, S., Bennett, C. L., \& Wolf, M. S. (2006). Social stigma concerns and HIV medication adherence. AIDS Patient Care STDS, 20(5), 359368.

Robertson, K., Parsons, T. D., Van Der Horst, C., \& Hall, C. (2006). Thoughts of death and suicidal ideation in nonpsychiatric human immunodeficiency virus seropositive individuals. Death Stud, 30(5), 455-469.

Roy, A. (2003). Characteristics of HIV patients who attempt suicide. Acta Psychiatrica Scandinavica, 107(1), 41-44.

Schneider, S. G., Taylor, S. E., Hammen, C., Kemeny, M. E., \& Dudley, J. (1991). Factor influencing suicide intent in gay and bisexual suicide ideators: differing models for men with and without human immunodeficiency virus. J Pers Soc Psychol, 61(5), 776-788.

Suppapitiporn, S., Thavichachart, N., \& Suppapitiporn, S. (2004). Social support in depressed patients who attempted suicide. J Med Assoc Thai, 87 Suppl 2, S266-271.

Thompson, M. P., Kaslow, N. J., Short, L. M., \& Wyckoff, S. (2002). The mediating roles of perceived social support and resources in the self-efficacy-suicide attempts relation among African American abused women. J Consult Clin Psychol, 70(4), 942-949.

Vanable, P. A., Carey, M. P., Blair, D. C., \& Littlewood, R. A. (2006). Impact of HIVRelated Stigma on Health Behaviors and Psychological Adjustment Among HIVPositive Men and Women. AIDS Behav.

Warner, J., McKeown, E., Griffin, M., Johnson, K., Ramsay, A., Cort, C., et al. (2004). Rates and predictors of mental illness in gay men, lesbians and bisexual men and women: Results from a survey based in England and Wales. Br J Psychiatry, 185, 479-485. 
Table 1: Socio-demographics, lifestyle and medical characteristics of participants in relation to suicide attempts - VESPA study $-\mathrm{n}=2,932$.

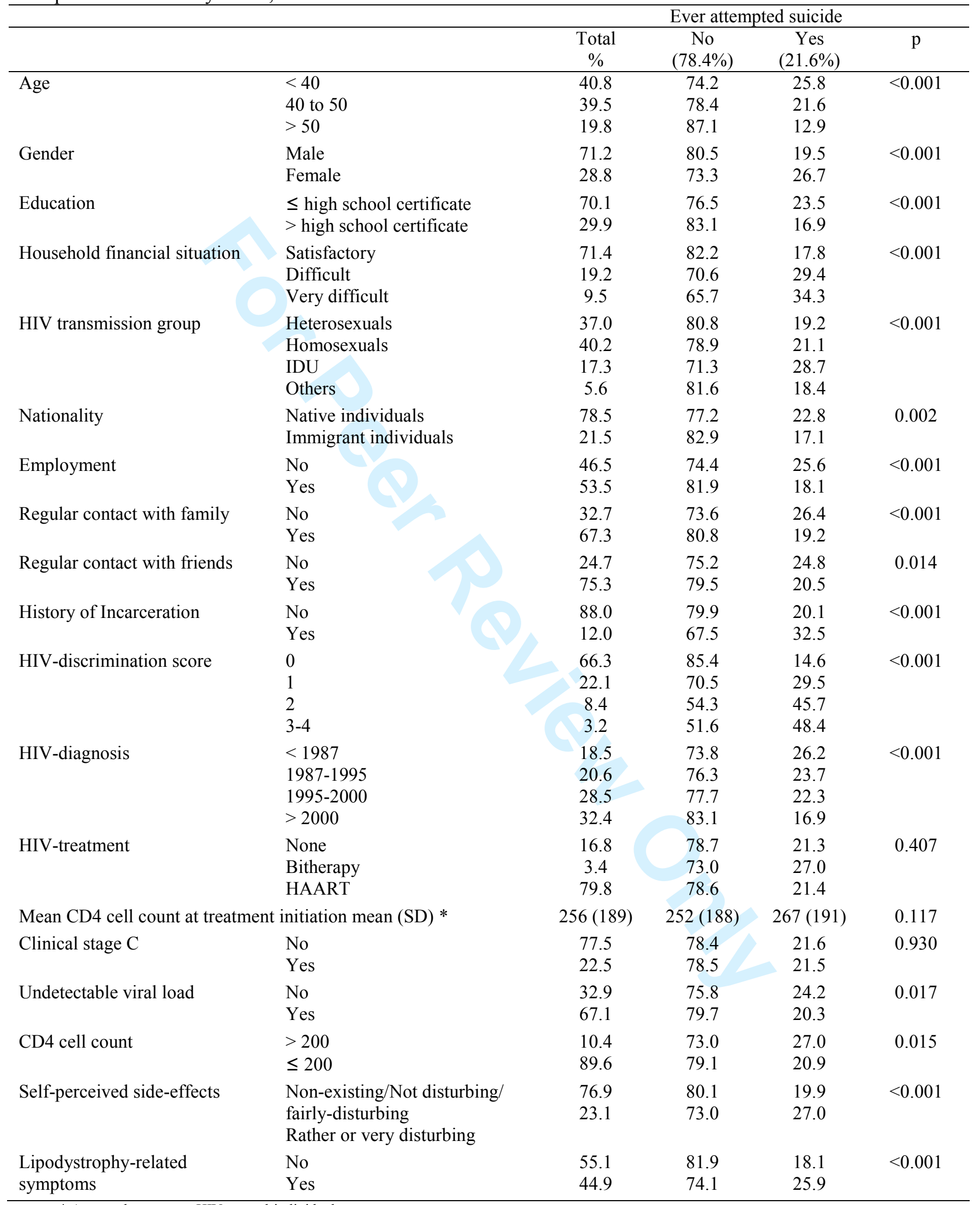

* Among then current HIV-treated-individuals. 
Table 2: Factors associated with suicide attempts - univariate and multivariate analysis VESPA study $-\mathrm{n}=2932$.

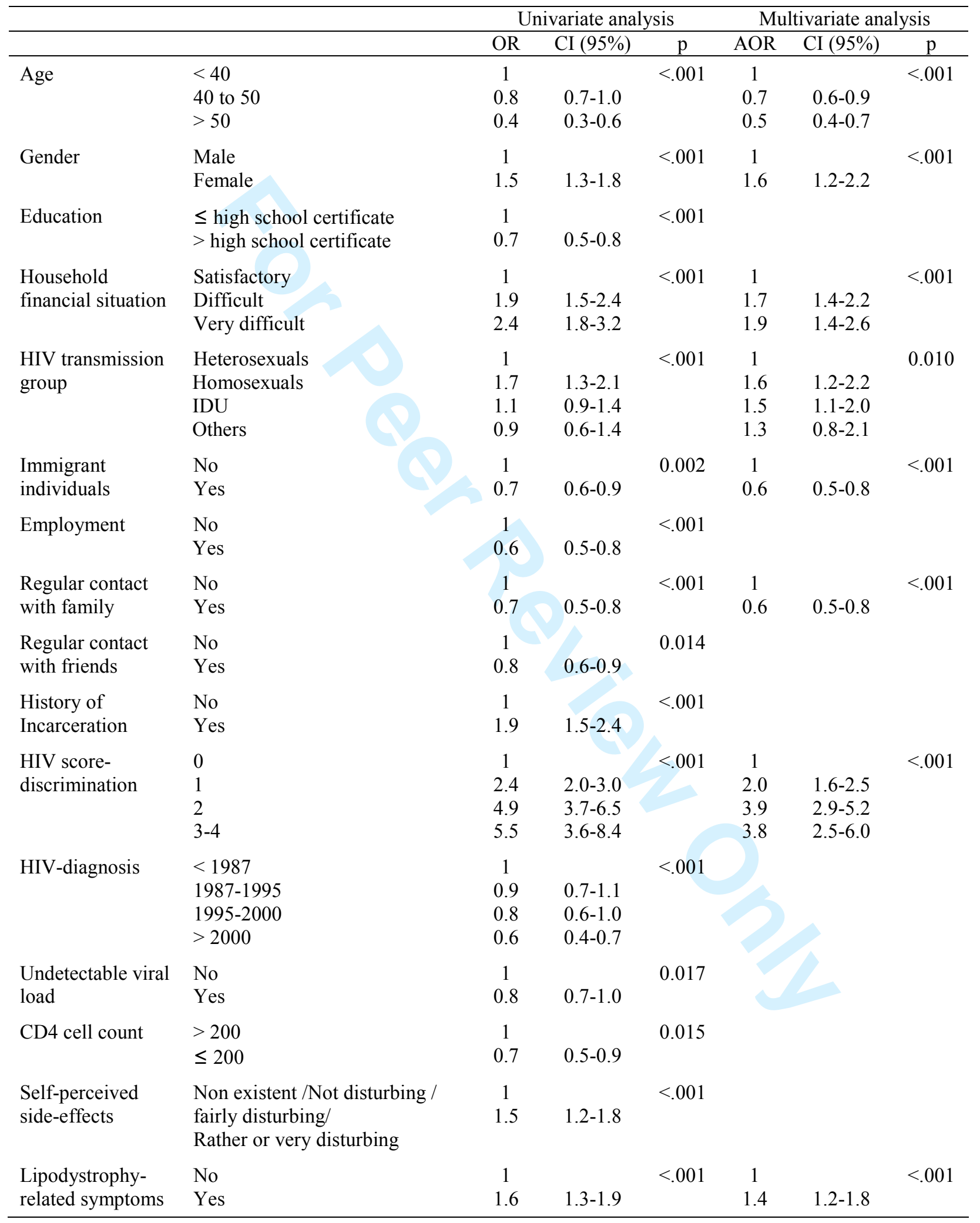




\section{Referee 1.}

Abstract:

HIV as well as AIDS should be written in full first before the abbreviations can follow.

People Living With HIV/AIDS (PLWHA) must be explained or stratified. This is very important as there is the need to understand at what stage of the HIV infection and/or full blown AIDS was the attempted suicide (AS) prevalence referred to.

HIV and AIDS have been written in full before the use of their abbreviations.

People living with HIV/AIDS is a commonly used expression referring to individuals HIVinfected whatever the stage of the infection. The VESPA survey was carried out on a sample of such individuals HIV in order to generate a picture of the epidemic in France in 2003. Consequently, our prevalence of SA refers to the whole HIV-infected population and not only to one part at a particular stage of the illness. Moreover, our report of SA is retrospective and does not take into account the severity of the illness at the time of the SA. However, the clinical parameters which enable documenting the severity of the infection, in particular the clinical stage (which indicates the worst stage of the infection in an individual's life) and the CD4 cell count at treatment initiation (which approximates the NADIR of an individual's CD4 cell count) are included in the analysis and the association between these variables and the outcome variable was tested. Moreover the proportion of patients at AIDS stage in the VESPA Survey and in the French National database was similar.

Please take note of some grammatical errors in the conclusion, including the seemingly omitted "with".

We agree with the reviewer and thank him for his suggestions in order to clarify the manuscript.

\section{Objective \& Methodology:}

There is concern about participants' selection bias and the actual reason for the 15 euro voucher given to participants was not given.

It is great that efforts seem to have been made to take care of some of these but the weighting procedure, as described in the study, does not look reproducible.

Population of the study area as well as the HIV/AIDS prevalence of the catchments' areas of the health institutions from which participants were drawn are not clearly stated. Generalizability of the results might therefore become questionable, especially with the 
wide scope of the heading of the article (Suicide attempts among people living with HIV in France).

A modification of the topic might become necessary.

The participants gave their written informed consent and were each compensated with a 15euro voucher after the study (patients were not aware of this payment when asked to participate). The following sentence has been added to clarify this. The participants gave their written informed consent and were each compensated with a 15-euro voucher after the study (patients were not aware of this payment when asked to participate).

Eligible criteria are indicated in the data collection paragraph: "Eligible subjects were outpatients at least 18 years of age and who had been diagnosed with HIV-1 at least 6 months previously. Those who were foreign citizens had to have been living in France for at least six months to be eligible." The complete sampling procedure is available in the previously published article (Dray-Spira, Spire, Heard, \& Lert, 2007; Peretti-Watel et al., 2005; Preau et al., 2007). The weighting procedure enabled the selection bias to be taken into account and to ensure the generalizability of the results to the whole HIV-infected French population being followed up in hospitals.

\section{Results}

Under the subheading suicide attempts $(S A)$, it is not clear if the $22 \%$ referred to include attempts made before HIV infection. The same applies to the headings on the two tables. The expression "lifetime" might have to be changed or defined in proper context.

The expression "lifetime" has been explained as follows: Participants were asked whether they had ever attempted suicide, either before or after HIV infection: once/several times/never. Documented SAs did not necessarily occur after HIV infection. We mentioned this point in the discussion as a limit of the study.

It is not clear if there are some people who AS before having HIV and had repeat of AS after the diagnosis of HIV. Also if there are people who had AS before HIV but never had a repeat of AS after the HIV diagnosis.

As explained in the limitations section, the nature of the data doesn't permit us to specify the time of AS (i.e. whether it occurred before or after the diagnosis of HIV).

In about the second paragraph, "regarding clinical" are presumably two words and should be so treated.

After re-examining the paper we found that both words were indeed separate. 


\section{Discussion}

Some grammatical corrections should be checked and the word "concern" does not look appropriate in the areas it was repeated.

We thank the reviewer and clarify this sentence as follows:

"More specifically, in France, $6 \%$ of males and 9\% of females in the general population have attempted suicide."

There are again some words that are written in combination that deserve separate existence, down to "mental health" in the closing sentence of the first paragraph.

The "finally" seems to have come too early in the first paragraph, only for other connecting words "in addition" to start another sentence down the line "---in addition, our results confirm that HIV-infected people ---“.

We agree with the reviewer and thank him to suggestions in order to clarify the manuscript. "Mental health" has been replace by "AS" and "finally" has been replace by "in addition".

\section{Conclusion}

This subheading did not come out in my download but there is a sign that suggests that it might be there.

As suggested, we added the subsection « Conclusion».

Some grammatical corrections might have to be done here as well. "Our study ---" and “our result ---" might have to be impersonalized. Some other such expressions before them should also be looked at.

We thanks the reviewer to these recommendations and we have change utilisation of "our" by some others impersonalized words such as "these results" or this study.

\section{Acknowledgement}

It is not clear as to the nature of support or contending interests the bodies mentioned have in the study.

The VESPA Group is constituted by the researchers who took part in the study. The ANRS is the financial support of the study.

Also, the VESPA group is the research team referred to in the Acknowledgements section but we do not decide the structures for expressions of acknowledgement. 


\section{Reference}

The reference need editing and should be set out properly (depending on the journal's format).

The references have been adapted to the journal's format.

Referee: 2

This is a manuscript on an important topic that adds knowledge to the field. There should be more recognition of and discussion of psychiatric diagnoses such as anti-social personality disorder, PTSD, addiction, and depression. These are all associated with elevated suicide risk, all prevalent among IVDA/HIV positive people, and can themselves contribute to perception of illness and stigmatization. I also would like to see more discussion of the limitations of the sample (only those in treatment, only about $40 \%$ asked to participate) and what that might imply. In reality, it is quite possible that the individuals in this study were actually higher functioning than those who were not in treatment which makes the prevalence of suicidal behavior more striking. It does not seem warranted to attribute the prevalence of suicidal behavior to HIV. Psychiatric issues that preceded infection also could account for suicidal behavior. While the prevalence of suicide attempts is higher than the population, it is actually quite a bit lower than that reported, for example, in HIV positive incarcerated women (Zlotnick, Lewis). This suggests that psychopathology, rather than serostatus, has a not surprisingly significant role. The authors should acknowlege that the absence of formal psychiatric diagnosis is a significant limitation of this study. The importance of high risk behavior, IVDA, past trauma and history of abuse should be mentioned. Overall, in a manuscript addressing suicidal behavior, there needs to be a stronger literature review of psychiatric antecedents of such behavior. HIV positive populations often have diagnoses that, in and of themselves, predispose to suicide.

We agree with the reviewer about the fact that psychopathology has a significant role on AS and AS is not completely due to serostatus. Moreover, the lack of information about psychopathology represents an important limitation, but it seems important to underline that this study aimed at identifying factors related to HIV status associated with HIV in order to give key information to health care providers to improve their follow-up.

In order to recognise this point, we added an explanation in the limitations section.

"Last but not least, there is a lack of data about prevalence of psychiatric disorders in this sample. The design of the study did not take into account information about the psychopathology of PLWHA reporting AS. This limitation is important due to the fact that literature on the subject has identified some psychopathological conditions such as post traumatic disorder or psychiatric disorder as being more likely associated with AS PLWHA who are likely to need particular psychiatric assessment, medical and mental health treatment 
(Ayuso Mateos, Lastra, \& Montanes, 1996; Nina A. Cooperman \& Jane M. Simoni, 2005; James, Rubin, \& Willis, 1991; Lewis, 2005; Roy, 2003). Nevertheless, this study aimed at identifying factors related to serostatus associated with AS in order to give some guidelines related to improving the follow-up of HIV infected people."

Dray-Spira, R., Spire, B., Heard, I., \& Lert, F. (2007). Heterogeneous response to HAART across a diverse population of people living with HIV: results from the ANRS-EN12VESPA Study. Aids, 21 Suppl 1, S5-12.

Peretti-Watel, P., Riandey, B., Dray-Spira, R., Bouhnik, A. D., Sitta, R., \& Obadia, Y. (2005). Comment enquêter la population séropositive en France ? L'enquête ANRS-EN12VESPA 2003. Population, 60(4), 525-550.

Preau, M., Marcellin, F., Carrieri, M. P., Lert, F., Obadia, Y., \& Spire, B. (2007). Healthrelated quality of life in French people living with HIV in 2003: results from the national ANRS-EN12-VESPA Study. Aids, 21 Suppl 1, S19-S27. 